\title{
Establishment of a Sentinel Laboratory-Based Antimicrobial Resistance Surveillance Network in Ethiopia
}

Carmen Hazim, Rajiha Abubeker Ibrahim, Matthew Westercamp, Gebrie Alebachew Belete, Berhanu Amare Kibret, Theresa Kanter, Getnet Yimer, Thuria Siraj Adem, Kurt B. Stevenson, Maritza Urrego, Kashmira N. Kale, Michael W. Omondi, Daniel VanderEnde, Benjamin J. Park, Michele M.B. Parsons, and Kathleen M. Gallagher

In 2014, as part of the Global Health Security Agenda, Ethiopia was provided the technical and financial resources needed to prioritize antimicrobial resistance $(A M R)$ in the national public health sphere. Under the direction of a multi-stakeholder working group, AMR surveillance was launched in July 2017 at 4 sentinel sites across the country. The AMR surveillance initiative in Ethiopia represents one of the first systematic efforts to prospectively collect, analyze, and report national-level microbiology results from a network of hospitals and public health laboratories in the country. Baseline readiness assessments were conducted to identify potential challenges to implementation to be addressed through capacity-building efforts. As part of these efforts, the working group leveraged existing resources, initiated laboratory capacity building through mentorship, and established infrastructure and systems for quality assurance, data management, and improved coordination. As a result, AMR surveillance data are being reported and analyzed for use; data from more than 1,700 patients were collected between July 2017 and March 2018. The critical challenges and effective solutions identified through surveillance planning and implementation have provided lessons to help guide successful AMR surveillance in other settings. Ultimately, the surveillance infrastructure, laboratory expertise, and communication frameworks built specifically for AMR surveillance in Ethiopia can be extended for use with other infectious diseases and potential public health emergencies. Thus, building AMR surveillance in Ethiopia has illustrated how laying the foundation for a specific public health initiative can develop capacity for core public health functions with potential benefit.

Keywords: Surveillance, Antimicrobial resistance, Global health security, Laboratory capacity building

Carmen Hazim, MPH, is an Epidemiologist; Matthew Westercamp, PhD, is an Epidemiologist; Michael W. Omondi, MS, is a Microbiologist; Daniel VanderEnde, MD, is Medical Officer; and Benjamin J. Park, MD, is Chief, International Infection Control Program; all in the Division of Healthcare Quality Promotion, US Centers for Disease Control and Prevention (CDC), Atlanta, Georgia. Michele M. B. Parsons, PhD, is a Microbiologist, Center for Global Health, Division of Global Health Protection, CDC, Atlanta. Rajiha Abubeker Ibrahim, MSc, is a Researcher, Clinical Bacteriology and Mycology Research Team, and Gebrie Alebachew Belete, MSc, is AMR Coordinator, Bacteriology, Parasitology, and Zoonosis Directorate; both at the Ethiopian Public Health Institute, Addis Ababa, Ethiopia. Berhanu Amare Kibret, MD, MPH, MBA, is Global Health Security-Technical Officer; Theresa Kanter, MURP, is Program Deputy; Kashmira N. Kale, MPH, MBA, is PHI/CDC Global Health Fellow; and Kathleen M. Gallagher, DSc, is Program Director; all in the Division of Global Health Protection, CDC, Addis Ababa, Ethiopia. Getnet Yimer, PhD, is East Africa Regional Director, Global One Health Initiative, Ohio State University, Addis Ababa, Ethiopia. Thuria Siraj Adem, MPH, is ASM Local Consultant for Ethiopia, International Affairs, American Society for Microbiology (ASM), Addis Ababa, Ethiopia. Kurt B. Stevenson, MD, is Professor of Medicine and Epidemiology, Department of Internal Medicine, College of Medicine, Ohio State University, Columbus, Ohio. Maritza Urrego, BBA, CPM, is Program Specialist, International Affairs, ASM, Washington, DC. 
$\mathrm{T}^{\mathrm{n}}$ he Global Health Security Agenda (GHSA) was launched through a partnership of more than 64 nations and international stakeholders in February 2014 to pursue multilateral and multisectoral approaches to strengthening the capacity of individual nations to prevent, detect, and respond to infectious diseases threats. GHSA has worked to elevate global health security as a national and global priority.

Antimicrobial resistance (AMR) is a global health threat that was recognized in the 2016 United Nations General Assembly call for action. ${ }^{1}$ Resistance occurs when microorganisms change to render an antibiotic ineffective; AMR pathogens can become epidemic, contribute to increased patient morbidity and mortality, and have substantial negative economic impacts. ${ }^{2}$ While AMR occurs in all countries, the burden of AMR and the emergence of novel resistance is disproportionately concentrated in low- and middle-income countries. ${ }^{3}$ However, the lack of microbiology capacity and public health surveillance infrastructure in many low- and middle-income countries limit the ability to detect, respond, and ultimately prevent AMR. To address this need, the GHSA prioritized combating antimicrobial resistance and establishing a national laboratory system to improve access to quality microbiology and help establish AMR surveillance systems. ${ }^{4}$

A 2009 report by the Drug Administration and Control Authority of Ethiopia concluded that, while data were limited, evidence suggested a high level of antibacterial resistance, and it called for a national approach to prevent further AMR development and spread in Ethiopia. ${ }^{5}$ Subsequent work identified limited microbiology laboratory capacity and capability and the lack of a systematic national AMR surveillance system to be principal limitations to effective AMR response in Ethiopia. ${ }^{6}$ The country was provided access to technical resources and funding mechanisms through GHSA to establish AMR surveillance and AMR control as national public health priorities.

Developing a surveillance system that can quantify and monitor AMR is expected to benefit the communities it serves by informing clinical decisions and helping to guide public health actions to effectively prevent and contain the spread of resistant pathogens. Additionally, the surveillance infrastructure, national leadership, laboratory expertise, and communication frameworks built specifically for AMR surveillance can be extended to the monitoring of other infectious diseases and can be valuable in an effective national response to many public health emergencies. This article describes how laboratory-based AMR surveillance was implemented in Ethiopia, discusses critical challenges, and provides lessons learned to help guide successful AMR surveillance in other settings.

\section{AMR Surveillance Working Group}

There are many different strategies for establishing public health surveillance of AMR. Surveillance can target microbial pathogens (microorganisms that have caused illness), commensals (microorganisms living in or on living things without causing illness), or organisms from the environment. In addition, strategies can be human-centric, animal-centric, or environmental (One Health approach). Efforts may focus on detecting and describing antibiotic treatment failures (pathogens that have survived and continued to cause illness despite appropriate antibiotic treatment), phenotypic susceptibility (organisms' inability to grow in a laboratory in the presence of carefully measured amounts of antibiotics), or genetic markers of resistance (bits of genetic information associated with resistance to different antimicrobials). Finally, surveillance systems can be designed to represent smaller well-defined populations, such as a single health facility; describe larger populations more generally, such as nations or states; or systems may simply strive to gain an understanding of circulating resistance without representing any defined population. Each strategy has strengths and limitations, and most systems ultimately represent a mix of strategies, but virtually all AMR surveillance requires accurate and reliable microbiology as the primary source of data.

In 2016, an AMR surveillance working group was formed in the Ethiopian Public Health Institute (EPHI), with support from the Food Medicine and Health Care Administration and Control Authority of Ethiopia (FMHACA). Technical assistance and coordination were provided through international partnerships with the US Centers for Disease Control and Prevention (CDC) Division of Global Health Protection and Division of Healthcare Quality Promotion. Contract support for specific AMR activities was provided by the American Society for Microbiology (ASM) and the Ohio State University (OSU) Global One Health initiative. The key objectives of the AMR surveillance working group were:

1. Develop a national AMR surveillance plan for Ethiopia.

2. Implement, in phases, national AMR surveillance in Ethiopia.

3. Ensure AMR surveillance data are of actionable quality.

4. Establish the infrastructure to transport isolates from surveillance sites for centralized confirmation testing and storage.

Implementation milestones achieved by the Ethiopian AMR surveillance working group are summarized in Figure 1.

\section{The National AMR Surveillance Plan}

Balancing available resources with a national focus on One Health AMR strategies embracing human, animal, and environmental sources, the AMR surveillance working group decided to start with a human-centric approach to allow the use of existing clinical laboratory services as the primary data source. The surveillance strategy that was 


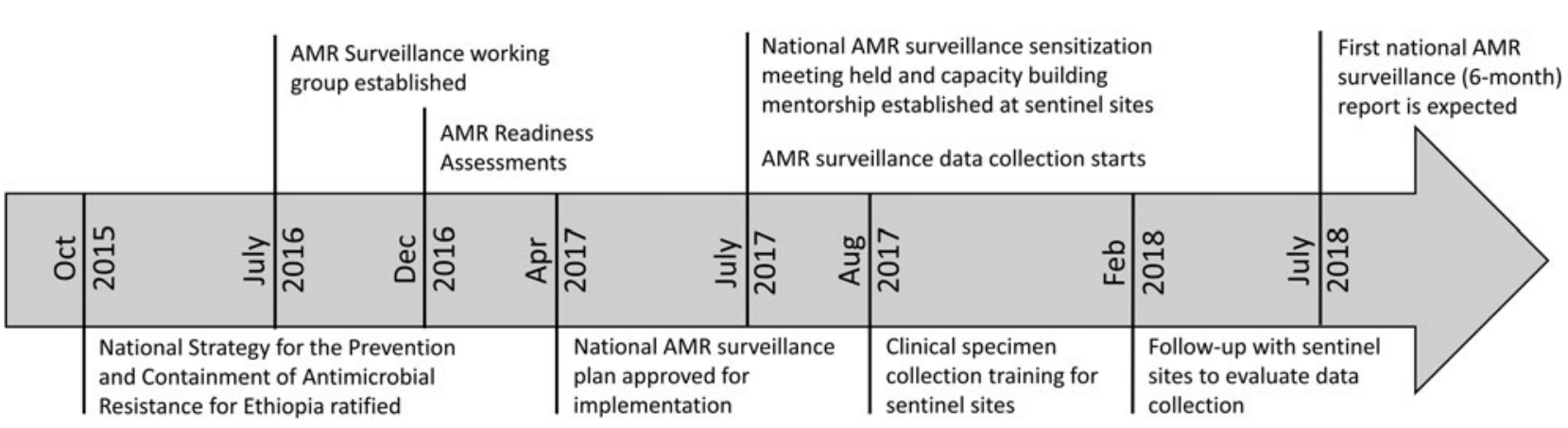

Figure 1. Implementation milestones for the Ethiopia AMR surveillance working group through July 2018

developed adhered to the guidance provided by the World Health Organization (WHO) Global Antimicrobial Resistance Surveillance System (GLASS) framework. AMR surveillance targeting animal and environmental sectors will be incorporated when those relevant data sources are available.

Surveillance efforts have initially focused on capturing patient and isolate data related to specimens routinely referred for microbiological evaluation. Because clinical laboratories in Ethiopia rely primarily on manual microbiology methods and molecular methods are infrequently performed, AMR surveillance was limited to phenotypic susceptibilities in clinically relevant pathogens. Data capture was limited to patients submitting a specific set of specimen types (ie, urine and pus) and targeted pathogens (ie, Escherichia coli, Klebsiella pneumonia, and Staphylococcus aureus). These specimen and pathogen sets were considered to be of local clinical and epidemiologic importance, required microbiology capacity, and were highlighted as important in GLASS. It is important to note that while surveillance efforts targeted a few pathogens, laboratories continued to test for all clinically relevant pathogens. As capacity increases, the surveillance program may be expanded to additional specimen and pathogen sets or to include linkage to infections and clinical syndromes that would provide data on the burden of AMR in Ethiopia.

The AMR working group devised a nodal sentinel surveillance structure because of the decentralized Ethiopian public healthcare system in which health sector authority is primarily held at the regional and district (woreda) levels. ${ }^{7}$ Based on regional needs and interest, regional- or districtlevel public health laboratories, which provide both reference and clinical testing services to a number of sentinel healthcare facilities, can serve as reporting nodes that interact directly with the AMR Surveillance National Coordinating Center (NCC). Additionally, higher functioning sentinel healthcare facilities with in-house microbiology services can operate independently of the regional- or district-level reporting nodes and can report directly to the NCC, which in turn can provide support and reference testing. As the NCC, the National Reference Laboratory (NRL) at EPHI supports implementation, ensures coordination, and helps facilitate resource allocation to all facility, regional-, and district-level reporting nodes. Figure 2 provides a diagram of possible reporting structures providing AMR surveillance data.

Following a comment and review period, the Ethiopian Federal Ministry of Health approved their National AMR Surveillance Plan for implementation in April 2017.

\section{AMR Readiness Assessment and Site SELECTION}

In order to target resources required for early implementation, AMR surveillance was initially established in a small select group of surveillance sites, including both directly reporting healthcare facility-level nodes and district- or regional-level reporting nodes, with improved national representativeness through phased expansion. Site selection started with the identification of laboratories currently processing clinical microbiology samples. Of the 35 laboratories identified, 16 were prioritized for implementation based on existing laboratory capacity, number of urine and pus samples submitted, and a willingness to participate. AMR readiness assessments were then conducted to evaluate relevant laboratory practices including specimen management, quality microbiological testing, and laboratory information management. Readiness assessments were performed using standardized tools developed by CDC's International Infection Control Program (IICP) and ASM. Assessments included direct observation, review of laboratory documentation, and interviews with staff and administrators.

Surveillance readiness assessments revealed that, despite clinical microbiology being conducted, improved laboratory capacity was needed to ensure the available data were consistently interpretable and useful in a surveillance context. In general, assessment findings identified the following gaps with the potential to affect data use:

- A lack of standardized procedures for quality assurance and quality control practice;

- Limited laboratory staff training and experience in microbiology;

- Limited access to reliable reagents and essential microbiology supplies and equipment; and

- Inefficient data management and reporting. 


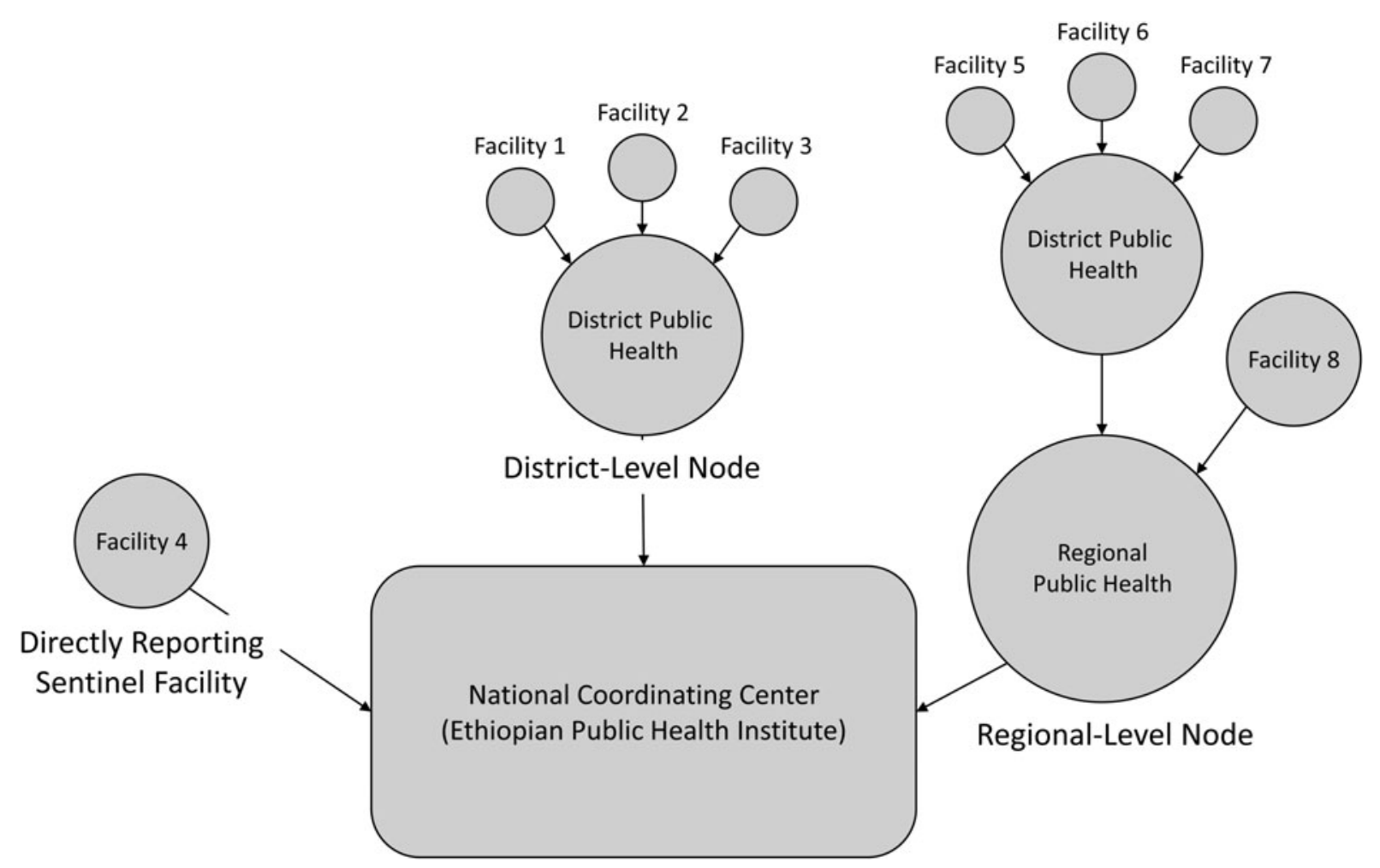

Figure 2. Diagram of the nodal reporting structures providing surveillance data to Ethiopia's national AMR surveillance system

The identified limitations led to a 3-phase approach for site implementation. Phase 1 includes 4 sites, phase 2 includes 6 sites, and phase 3 includes 6 additional sites. Implementation phase assignment was based on current laboratory capacity, readiness to participate, and geographic location, with the goal of efficient targeting of laboratory capacity building and more rapid surveillance implementation.

\section{Mentorship and Laboratory Capacity BUILDING}

Following the identification of phase 1 sites, repeat site visits were conducted to specify laboratory deficiencies, determine resources available, and collaborate with laboratory staff to establish a 1-year capacity building work plan for each site, including the NRL. The hallmark of all these work plans was the establishment of individual laboratory mentorship led by ASM. While costly, direct 1-on-1 mentorship provided the individualized guidance required to address the many types of issues made evident by the readiness assessments. Mentorship helped identify and leverage individual site strengths and promote creative ad hoc solutions to challenging issues. Lastly, in place of a rigid set of requirements for participation, site mentors were able to recommend sites for inclusion based on ongoing process improvement resulting in more rapid surveillance scale-up.

Laboratory mentorship involved quarterly onsite visits from experienced microbiologists for a minimum of 2 weeks. Mentorship visits were coordinated by EPHI-NRL and an ASM consultant with local expertise in project management and microbiology, using tools and materials developed by working group members. These materials included antibiotic panels and standard operating procedures (SOPs) aimed at standardizing microbiology and reporting methods. All mentored sites were enrolled in an internationally recognized external quality assurance (EQA) program to help monitor and quantify improved capabilities over time. In addition, sites were provided with relevant quality control (QC) strains as needed and licensed Clinical and Laboratory Standards Institute (CLSI) guidelines for results interpretation and reporting.

The short-term objective of laboratory capacity building was to move enrolled sites toward providing reliable surveillance data, with an ultimate goal of achieving international accreditation in microbiology. As of June 2018, the NRL had attained international ISO 15189;2012 accreditation, and 1 additional phase 1 site was preparing to be assessed for accreditation. To help ensure laboratory quality improvement efforts are sustained, EPHI-NRL has been gradually capacitated to take leadership of the laboratory mentorship activities. EPHI-NRL staff accompany and support the ASM staff during quarterly mentorship visits and have begun to conduct independent interim site visits to meet this objective.

\section{Microbiology Supplies and Equipment}

Purchasing and inventory of supplies and equipment has been identified as a long-standing limitation for clinical 
microbiology in many low- and middle-resource settings. ${ }^{8}$ The lack of a full complement of reagents, standard microbiology supplies, and the equipment required for culture, identification, and antibiotic susceptibility testing was a critical limiting factor at all sentinel sites. Due to the need to ensure timely availability of essential supplies and equipment for rapid surveillance scale-up, the working group created a list of essential bacteriology reagents and supplies and obtained a 6-month to 1-year supply for each sentinel site from established international sources. For long-term sustainability, this essential list was used to negotiate with the central procurement agency of Ethiopia to help ensure uninterrupted availability moving forward. Laboratory equipment was assessed at each site and was replaced or repaired as needed. Additionally, a system to capture and document issues pertaining to the quality and availability of supplies was established to improve communication between laboratories and allow more coordinated response to supply issues.

\section{Clinical Sensitization and Specimen Management}

To support efforts to increase laboratory capacity, a series of semi-structured clinical assessments led by Ohio State University examined specimen management and current diagnostic microbiology stewardship practices (the ordering, performing, and reporting of diagnostic tests). Issues identified through these clinical assessments fit into 4 thematic categories:

- Low utilization of microbiology services

- Variable quality of clinical specimens

- Specimen collection restricted to patients failing empiric antibiotic treatment

- Common preadmission antibiotic use limiting utility of microbiological testing

A combined surveillance introduction for key clinicians and training workshop on microbiology clinical specimen collection best practices was developed. The training emphasized the importance of appropriate sample collection for use by healthcare facilities and the national AMR surveillance initiative. For long-term sustainability, training materials and job aids supporting the ongoing training of frontline clinical staff were developed. An annual training of trainers program targeting facility-level training staff was established to provide guidance in using the developed materials and to promote effective methods of adult learning.

\section{National Coordinating Center AND REFERENCE LABORATORY}

An unintended consequence of Ethiopia's decentralized public health laboratory system has been the underuse of the national bacteriology reference laboratory at EPHI. The nodal sentinel surveillance strategy Ethiopia selected for AMR surveillance requires substantial centralized capacity to provide system coordination, ensure data quality, coordinate data management, and for dissemination and use of results. The national bacteriology reference laboratory at EPHI needed additional assistance to fulfill their vision of being a national coordinating center for AMR surveillance. EPHI participated in early efforts to train and redistribute staff, sought laboratory mentorship, established formal and informal lines of communication with surveillance sites, and improved the supply systems within the AMR surveillance sentinel network. Their efforts were ultimately successful in preparing the EPHI-NRL to fulfill its role as the national coordinating center for AMR surveillance. To ensure long-term sustainability of the AMR surveillance and laboratory mentorship programs, the NRL is continuing to advocate for dedicated staff and resources from EPHI and ministry of health leadership.

Due to a lack of established procedures and the logistics required for specimen and isolate transport, bacteriology at EPHI-NRL had limited opportunity to engage in reference (providing testing for unknown isolates sent from limited capacity laboratories) or confirmation testing (retesting isolates for results comparison). An innovative but limited public-private partnership between EPHI and the Ethiopian postal service had successfully implemented specimen referral and transport of HIV and TB isolates within a nationwide laboratory network. ${ }^{9}$ Expansion of this program to allow transport of the bacterial isolates and clinical specimens to support AMR referral and confirmation testing are planned with preliminary testing under way.

\section{Data Management and Analysis}

When AMR surveillance began, none of the phase 1 sites used a digital laboratory information management system for microbiology. Evaluations of the laboratory data flow at each sentinel site revealed varied paper-based data management with no consistent methodology for AMR surveillance data capture. Small pilot projects exploring the integration of WHONET software for surveillance site data entry were initially challenging because of the perceived disruption to existing workflow and increased workload. Ultimately, individualized solutions for data entry were created to ensure data collection was integrated with existing practices and was acceptable to laboratory staff and management. Data collection was ultimately accomplished by establishing a site-specific digital WHONET database at each site but allowing paper-based data capture based on existing laboratory forms with final database entry facilitated by the NCC. While not required by the system, sentinel site staff were provided basic training on digital data entry, data management, and data analysis to promote digital collection and the use of microbiology data in the future. 
All data analyses are performed at the NCC using the freely available WHONET software and a spreadsheet program (eg, Microsoft Excel) as needed for improved visualization of results. Site-specific analyses, including calculation of hospital-level patterns of resistance and antibiograms are completed and periodically returned to individual sites as the number of tested isolates allows. Anonymized aggregate results will be used for national and international AMR reporting. However, the data systems at sentinel sites and at the NCC will continue to be reevaluated, and improvements may be needed.

\section{Initial AMR Surveillance Findings}

As of March 2018, all 4 phase 1 surveillance sitesincluding 2 healthcare facility-level nodes, 1 regional-level node servicing multiple healthcare facilities, and the clinical testing performed at EPHI in support of several local healthcare facilities-were reporting AMR surveillance data. From July 2017 to March 2018, the system received patient and isolate data on 1,786 urine and pus specimens, collected from 1,767 patients. Sites providing sufficient data have been provided with preliminary antibiograms, and surveillance activities and findings are individually discussed with each site during regular NCC-led calls and site visits. The first formal AMR surveillance report is expected in July 2018. The government plans to promote use of these surveillance results to develop facility-level cumulative antibiograms, guide national stewardship policies, and continue to improve quality of microbiology services that have a direct impact on patient care.

The initial procurement of microbiological supplies was received and dispensed to the sentinel sites, and all have an active ongoing mentorship with ASM. Due to inconsistencies in performance of baseline EQA testing, we are still unable to quantify improvement; however, EQA performance has been systematized with corrective work plans based on results provided following each round. Changes in the number or quality of submitted clinical microbiology specimens to site laboratories has not been measured because of logistical challenges. Qualitatively, early engagement with clinicians at the sentinel sites has resulted in continued engagement around both laboratory use and the importance of appropriate specimen collection. The training of trainers program designed to provide a model of sustainable facility-level specimen collection training for clinical staff was developed based on needs expressed through this initial engagement.

\section{BUILDING ON SUCCESS}

The AMR surveillance initiative in Ethiopia represents one of the first systematic efforts to prospectively collect, analyze, and report national-level microbiology results from a network of healthcare facilities and public health laboratories in the country.

Developing a common vision for AMR surveillance allowed public health leaders throughout the Ethiopian public health system to constructively discuss and debate current limitations in laboratory infrastructure, microbiology supply procurement, laboratory data quality, and the far-reaching implications of the lack of information technology and digital laboratory information management systems. Through the implementation of AMR surveillance, GHSA provided access to the funding and the technical expertise needed to sustainably address some of these issues, thus building public health capacity through the performance of core public health functions. Following the successful initiation of AMR surveillance, the Ethiopian AMR working group identified several potential areas for growth:

- Expansion of surveillance activities to additional sentinel sites

- Establishment of bacterial isolate and specimen transport between peripheral laboratories and the reference laboratory at EPHI

- Exploration of alternative AMR surveillance strategies, including an isolate-based referral system in which participating laboratories submit presumptively identified isolates for confirmation of culture identification and antibiotic susceptibility testing; and/or time-limited surveillance, where laboratory capacity is temporarily augmented to provide reliable quantification of resistance over relatively short time periods

While substantial work remains, the implementation of AMR surveillance in Ethiopia has already provided public health tools and resources with potential value in national emergency response. The nodal sentinel surveillance structure itself provides a new framework embracing both direct healthcare facility reporting as well as information flow along established hierarchical public health laboratory structures. The surveillance infrastructure and relationships developed should facilitate the otherwise difficult establishment of the ad hoc disease monitoring and information systems that are needed for rapid response. Additionally, the increases in microbiology capacity have begun to create a system of laboratory "centers of excellence" with increased access to high-quality microbiology supplies, equipment, and training that are now available for national laboratory surge capacity.

Finally, the communication networks and regular systematic reporting naturally established through surveillance implementation allows opportunities for astute laboratorians and clinicians to report small problems to public health authorities before they come large public health emergencies. In short, building AMR surveillance in Ethiopia provides an example of how laying the foundation for a specific public health initiative has developed capacity for 


\section{AMR SURVEILLANCE INITIATIVE IN ETHIOPIA}

core public health functions with far-reaching potential benefits for global health security.

\section{REFERENCES}

1. World Health Organization. Draft resolution: Political Declaration of the high-level meeting of the General Assembly on antimicrobial resistance. September 22, 2016. https:// digitallibrary.un.org/record/842813/files/A_71_L-2-EN.pdf. Accessed August 22, 2018.

2. Toner E, Adalja A, Gronvall GK, Cicero A, Inglesby TV. Antimicrobial resistance is a global health emergency. Health Secur 2015;13(3):153-155.

3. Review on Antimicrobial Resistance. Tackling Drug-Resistant Infections Globally: Final Report and Recommendations. 2016. https://amr-review.org/sites/default/files/160518_Final\% 20paper_with\%20cover.pdf. Accessed August 22, 2018.

4. Centers for Disease Control and Prevention. Global Health Security Agenda: Action Packages. CDC website. Updated January 21, 2016. https://www.cdc.gov/globalhealth/security/ actionpackages/default.htm. Accessed August 22, 2018.

5. Drug Administration and Control Authority of Ethiopia. Antimicrobials Use, Resistance and Containment Baseline Survey Syntheses of Findings. Addis Ababa, Ethiopia; 2009. http:// apps.who.int/medicinedocs/documents/s18057en/s18057en.pdf. Accessed August 22, 2018.
6. Moges F, Endris M, Mulu A, et al. The growing challenges of antibacterial drug resistance in Ethiopia. J Glob Antimicrob Resist 2014;2(3):148-54.

7. Federal Democratic Republic of Ethiopia Ministry of Health. Health Sector Development Program IV: 2010/11-2014/15. October 2010. http://www.nationalplanningcycles.org/sites/ default/files/country_docs/Ethiopia/ethiopia_hsdp_iv_final_ draft_2010_-2015.pdf. Accessed August 22, 2018.

8. Barbé B, Yansouni CP, Affolabi D, Jacobs J. Implementation of quality management for clinical bacteriology in low-resource settings. Clin Microbiol Infect 2017; 23(7):426-433.

9. Kebede Y, Fonjungo PN, Tibesso G, et al. Improved specimen-referral system and increased access to quality laboratory services in Ethiopia: the role of the public-private partnership. J Infect Dis 2016;213(Suppl 2):S59-S64.

Address correspondence to: Carmen Emily Hazim, MPH

Epidemiologist International Infection Control Program Division of Healthcare Quality Promotion (DHQP) Centers for Disease Control and Prevention Atlanta, GA

Email: chazim@cdc.gov 